\title{
What's New on Alzheimer's Disease? Insights From AD Mouse Models
}

Christophe C Rey, Vanessa Cattaud, Claire Rampon*, and Laure Verret ${ }_{*}$, Research Center on Animal Cognition, Center for Integrative Biology, Toulouse University, France and CNRS, UPS, France

(C) 2019 .

Email address: laure.verret@univ-tlse3.fr(L. Verret)

\section{Introduction}

Design of Alzheimer's Disease Mouse Models

First Drug Trials Based on Preclinical Studies With AD Models: Targeting A $\beta$ Species

Alzheimer's Disease Patients Also Present Changes in Their Brain Activity

Hypersynchrony and Epilepsy in Alzheimer's Disease Mouse Models and Patients

Neuronal Mechanisms of Hypersynchrony/Epilepsy in Alzheimer's Disease Mouse Models

Using Antiepileptic Drugs Against Alzheimer's Disease?

Alteration of Brain Oscillations in Alzheimer's Disease Mouse Models and Patients

Conclusion

References

Further Reading

Relevant Websites

\section{Nomenclature}

AD Alzheimer's disease

AED Antiepileptic drug

APP Amyloid precursor protein

Aß Beta-amyloid

CSF Cerebrospinal fluid

DMN Default mode network

E/I Excitation/inhibition

EEG Electroencephalogram

FAD Familial forms of Alzheimer's disease

GSI Gamma secretase inhibitor
GSM Gamma secretase modulator

LEV Levetiracetam

MCI Mild cognitive impairment

NSAID Nonsteroidal antiinflammatory drug

PDGF $\beta$ Platelet derived growth factor $\beta$

PET Positron emission tomography

PS1 Presenilin-1

PS2 Presenilin-2

PV Parvalbumin

SOM Somatostatin

VGSC Voltage-gated sodium channel

\section{Introduction}

Aging goes with cognitive decline, which sometimes results from pathological insults. Among these insults, Alzheimer's disease (AD) is nowadays certainly the most prevalent neurodegenerative disease among the aging population worldwide. AD was first described by Dr. Alois Alzheimer in 1906. His description included various behavioral disruptions such as memory deficits, disorientation, aphasia and

\footnotetext{
* Shared-authorship.
} 
unpredictable behaviors. At the anatomical level, AD patients were found to exhibit a thinning of the cerebral cortex, associated with a widening of the lateral ventricles. Moreover, at the neuropathological level, Dr. Alzheimer reported the presence of extracellular plaques, now known to be formed by the aggregation of the beta-amyloid $(A \beta)$ peptides. Another hallmark of the disease is the formation of neurofibrillary tangles due to the hyperphosphorylation of Tau proteins inside the neurons. Whether and how these anatomical markers can be linked to the associated cognitive deficits observed in AD patients remain key questions to tackle. In this context, the generation and use of mouse models have proven to be highly relevant for testing hypotheses about the physiopathological mechanisms leading to the development of AD. Over the last decades, these models have guided a myriad of research efforts including preclinical studies and clinical trials. However, the successive failures of clinical trials that were designed based on these models, have today precipitated their calling into question. In this article, we present an overview of how these models have contributed to the understanding of the disease, highlighting their limitations but also emphasizing the new and still open opportunities they have provided.

\section{Design of Alzheimer's Disease Mouse Models}

There are two types of AD: the familial and the sporadic forms. The sporadic form developed by the vast majority of AD patients, about $95 \%$ of the cases, is late-onset, and has a multifactorial origin. The remaining $5 \%$ of AD cases exhibit one of the early-onset familial forms of AD (FAD). All FAD cases are caused by either autosomal dominant genetic mutations of APP, PSEN1, or PSEN2 genes (Gotz et al., 2018). All of these mutations affect genes involved in the proteolytic pathway of the Amyloid Precursor Protein (APP), resulting in the overproduction of A $\beta$ peptides. Today, more than 33 FAD-mutations have been described on the APP gene. PSEN1 and PSEN2 genes code for presenilin-1 (PS1) and -2 (PS2), respectively. These proteins are part of the enzymatic complex of the gamma-secretase, which is also involved in the production of $\mathrm{A} \beta$. Indeed, postmortem observations of the brain of both sporadic AD or FAD patients show a strong accumulation of amyloid $\beta$ peptides. These observations led Hardy and Higgin to expose in 1992 the amyloid cascade hypothesis stating that $A \beta$ accumulation is at the origin of the disease, from neuronal dysfunction to dementia (Hardy and Higgins, 1992).

Mouse models of $\mathrm{AD}$ are designed based on this amyloid cascade hypothesis. With age, wild-type mice never develop spontaneous AD-like pathology. In fact, murine APP produces lower levels of A $\beta$ and has a lower aggregation capacity even after overexpression of APP (Jankowsky et al., 2007). In contrast, introducing into the mouse genome one or several human APP, PSEN1, or PSEN2 genes harboring one or several FAD-linked mutations results in high levels of $\mathrm{A} \beta$ peptides associated with changes resembling AD pathology. Thus, mice harboring FAD-linked mutations turn out to exhibit symptoms and signs of the disease. We present below the transgenic lines that have been the most studied because they recapitulate much of the amyloid pathology seen in human AD.

The first transgenic AD mouse model, the PDAPP mouse, was reported in 1995 (Games et al., 1995). These mice overexpress the human $A P P$ gene (hAPP) harboring the Indiana mutation involved in FAD. As a result, they produce high $\mathrm{A} \beta$ levels from early age on, and display cognitive deficits including recognition memory, working memory (Dodart et al., 1999) and spatial memory (Daumas et al., 2008).

The same year, the Tg2576 model was developed. This model accounts among the most studied AD models during the past two decades (Hsiao et al., 1995). Tg2576 mice express the human $\mathrm{APP}_{695}$ gene carrying the FAD-linked Swedish double-mutation $\left(\mathrm{APP}_{\text {swe }}\right.$ ). Overexpression of this mutated $\mathrm{h} A P P$ induces a slow progression of the pathology comparable to that observed in human patients with sporadic $\mathrm{AD}$, thus allowing to study biological markers at early stages of the pathology. Tg2576 mice develop virtually no neurofibrillary tangles nor significant neuronal loss, and could indeed be considered as a partial model of AD (Westerman et al., 2002). The age-dependent increase of $A \beta$ in the brain of these mice results in the establishment of extracellular amyloid plaques in the cortex and hippocampus at 10-12 months of age (Kawarabayashi et al., 2001). At 18 months of age, these plaques are surrounded by neuronal loss, reactive astrogliosis and hyperphosphorylated tau. At the behavioral level, deficits in spontaneous alteration task and spatial memory were reported in Tg2576 mice by 3 months of age, i.e., long prior to amyloid plaques formation, and progressive age-related cognitive impairments are observed thereafter (King et al., 1999). The Tg2576 model is one of first mouse line which allowed demonstrating that while amyloid plaques define $\mathrm{AD}$, they correlate poorly with the cognitive impairments associated with $\mathrm{AD}$. Instead, it has been demonstrated that the presence of high levels of soluble $A \beta$ species is responsible for memory deficits in $\operatorname{Tg} 2576$ mice (Lesne et al., 2006).

Another frequently studied mouse transgenic line is the hAPPJ20 (Mucke et al., 2000). These mice also combine the Swedish and Indiana mutations of the human $A P P$ gene, but its expression is regulated by the platelet-derived growth factor $\beta$ (PDGF $\beta$ ) promoter. Amyloid deposition occurs at 5-7 months of age in the hippocampus and neocortex of hAPPJ20 mice, which exhibit age-related formation of A $\beta$ plaques starting at 8-10 months of age (Mucke et al., 2000). Aberrant behaviors in hAPPJ 20 mice such as locomotor hyperactivity and impairments in the object recognition task, have been reported as early as 2-3 months of age (Harris et al., 2010). Interestingly, cognitive impairments of hAPPJ20 mice have been correlated to the reduction of hippocampal neuronal activity, reflected by a depletion of calbindin (Palop et al., 2003). In such, hAPPJ20 mice allowed for the first time to link A $\beta$ accumulation with impaired neuronal activity, pointing out the AD-linked mechanisms of cognitive alterations (Palop et al., 2003).

While a large number of transgenic mouse models of AD bear one or several APP mutations, PS1 mutations are the most commonly recognized causes of early-onset FAD in humans. In fact, PS1 mutations cause APP processing to be shunted toward amyloidogenic pathway (Vetrivel et al., 2006) (Fig. 1). While transgenic mice expressing human presenilin variants display no overt pathology, they present the advantage of substantially accelerating and potentiating plaque deposition once crossed with APP transgenic lines. For example, the APPswe/PS1dE9 mice express the Swedish mutation of APP and a secondary transgene, the exon 9 deletion of PS1 



Fig. 1 Schematic illustration of the amyloidogenic and nonamyloidogenic pathways. (Center) The amyloid- $\beta$ precursor protein (APP) is the target of serial proteolytic cleavages. (Left) The $\alpha$-secretase is implicated in the neuroprotective, nonamyloidogenic pathway. Its cleavage of APP generates sAPP- $\alpha$ and the $\mathrm{C} 83$ fragment (83-residue C-terminal APP). The proteolysis of the C83 fragment by the gamma-secretase releases the APP intracellular (AICD) and a short fragment called P3. (Right) In the amyloidogenic pathway, the $\beta$-secretase cleaves APP to generate sAPP- $\beta$ and the C99 fragment (99-residue C-terminal APP). Then, the cleavage of C99 by the gamma-secretase produces the A $\beta$ fragment and the AICD. The accumulation of $\mathrm{A} \beta$ leads to its oligomerization and later to its aggregation into amyloid plaques.

(PS1dE9) (Jankowsky et al., 2001). In APPswe/PS1dE9 mice, the first amyloid plaques appear between 4 and 6 months of age (Jankowsky et al., 2004), and early cognitive disorders are reported at 3 months of age (Vegh et al., 2014).

Several other transgenic mouse models carrying one or several FAD-linked mutations have been generated in order to study the development of the pathology within a relatively short time frame. Most information about the characterization of selected AD mouse models is available (https://www.alzforum.org/research-models:alzheimers-disease).

Although none of these transgenic mice recapitulates the full spectrum of progressive neuropathological and behavioral changes observed in the human disease, they all show age-dependent amyloid deposition and memory loss. In such, they represent valuable in vivo systems to understand the neurobiological basis of AD pathology. Depending on the mutations, the promotor used for the expression of the transgenes, and depending on their number of copies, these mouse models exhibit different time course of the disease. However, they all have increased levels of soluble $A \beta$ and amyloid plaque formation associated with age-dependent cognitive deficits.

Over the past decades, these mice have provided strong evidence for the primacy of $A P P$ expression and $\mathrm{A} \beta$ deposition in $\mathrm{AD}$ pathology. Importantly, these animal models have also been relevant tools to evaluate whether pharmacological drugs known to reduce $A \beta$ production and/or reduce its neurotoxicity in vitro, could also be efficient in vivo, a critical step before advancing into clinical trials with AD patients. 


\section{First Drug Trials Based on Preclinical Studies With AD Models: Targeting Aß Species}

The amyloid cascade hypothesis identified the peptide A $\beta$ as a key player of AD. Studying how A $\beta$ is secreted from APP (Fig. 1) has led to the identification of molecular targets for the design of candidate drugs against $A \beta$ species. Thus, antibodies directed against $A \beta$ were designed in an attempt to directly neutralize the peptide. Remarkably, these drugs turned out to be promising in preclinical trials.

Indeed, the first immunization with anti-A $\beta$ antibodies was performed on 6-week-old PDAPP mice, i.e., before the onset of the disease, and PDAPP mice at 11 months of age, when they exhibit strong cognitive deficits and amyloid plaques (Schenk et al., 1999). It was found that immunization against $A \beta$ in young mice basically prevented age-dependent amyloid deposition, while late immunization significantly reduced levels of soluble $A \beta$ levels as well as the amyloid plaque load.

Following this pioneer study, two other groups also performed immunization against A $\beta$ peptide using the APPswe/PS1dE9 (Morgan et al., 2000) and TgCRND8 (Janus et al., 2000) mouse models of AD. In both studies, they reported a halving of the amyloid burden in the brains of immunized mice, associated with a significant improvement of mice cognitive performances, even at advanced stages of the pathology. Altogether these findings supported the hypothesis that abnormal A $\beta$ processing is decisive for AD pathogenesis. Based on these promising data, the first trials in humans were then launched.

Starting in 2000, a phase 1 clinical trial for AN1792 (synthetic Aß42 with QS-21 adjuvant) demonstrated tolerability and a positive response to immunization in patients (Bayer et al., 2005). The study also stated the safety of AN1792 and the need to enroll larger cohorts of patients to reliably determine the efficacy of the treatment. Hence, a phase 2 study was initiated to evaluate the efficacy of the immunization with AN1792. However, this clinical trial was suspended in 2002, after $6 \%$ of immunized patients developed meningoencephalitis (Orgogozo et al., 2003). Following the failure of this phase 2, the subjects engaged in phase 1 were included into a follow up study designed to evaluate the potential long-term effects of $A \beta$ immunization in AD patients. Interestingly, a significant reduction of the amyloid plaque load was found in the immunized AD patients. However, no evidence of cognitive nor survival improvement was found, suggesting that removal of amyloid plaques may not be enough to stop AD progression (Holmes et al., 2008). In parallel, 4.6 years after AN1792 immunization, responder patients exhibited a reduced cognitive decline compared to patients receiving placebo, and no cases of encephalitis were reported (Vellas et al., 2009). This study supported the idea that $A \beta$ immunization induced long term cognitive benefits in AD patients.

Other anti-A $\beta$ strategies have been tested. For instance, Aducanumab (BIIB037), which binds fibrillary and soluble A $\beta$ with high selectivity, was administrated to 22-month-old Tg2576 mice, reflecting an advance stage of the pathology. A very significant reduction of soluble and insoluble $A \beta$ was observed in these mice, as well as a drastic decrease of A $\beta$ plaque load (Sevigny et al., 2016). In the phase 2 of the clinical trial, Aducanumab was demonstrated to reduce $\mathrm{A} \beta$ load in AD patient according to a dose- and time-dependent manner (Sevigny et al., 2016). Amyloid plaque reduction was associated with a cognitive stabilization of treated patients, with a tendency toward amelioration (Sevigny et al., 2016). A phase 3 clinical trial was developed to assess the therapeutic effect of Aducanumab for early AD, giving hope for this therapeutic approach. However, the clinical trial had to be stopped because of the lack of efficacy in the interim analysis compared to the expected results.

Other strategies have consisted in decreasing soluble $A \beta$ and amyloid plaque in AD patients. Several preclinical studies have demonstrated the efficacy of gamma-secretase inhibitors (GSI) to decrease the proteolytic cleavage of APP into A $\beta$. For instance, the use of LY-411575 in Tg2576 (Das et al., 2012) and in APPswe/PS1dE9 mice (Garcia-Alloza et al., 2009) lowered the production of soluble $\mathrm{A} \beta$ and reduced amyloid plaque formation. However, the beneficial effect of this GSI is observed only when administrated during the early stages of the disease, hence before amyloid deposition. Another GSI, the Semagacestat was tested in AD patients with the aim to target later stages of the disease. However, Phase 3 of Clinical Trial 3 did not demonstrate any beneficial effects on cognition and higher doses resulted in serious side effects, including a worsening of functional abilities, skin cancer and infections (Doody et al., 2013). These side effects were likely caused by the interruption of the other physiological functions of gamma-secretases. Indeed, beside their role in the cleavage of APP, gamma-secretases are involved in the cleavage of the Notch protein that regulates cell proliferation, differentiation and cell growth (Kopan and Ilagan, 2009). Whilst gamma-secretase inhibitors did not keep promises in clinical studies, epidemiological studies showed a reduced incidence of AD among steady users of nonsteroidal antiinflammatory drugs (NSAIDs) have allowed the identification of gamma-secretase modulators (GSM) as putative drug candidates for the treatment of AD. Indeed, it was found in vitro and in vivo that some NSAIDs lower $A \beta 42$ production in favor of less toxic nonaggregating $A \beta$ species (e.g., A $\beta 37$, A $\beta 38$ ) by modulating gamma-secretase activity and without affecting other APP processing pathways or Notch cleavage (Weggen et al., 2003). The ability of these compounds to reduce A $\beta 42$ selectively has raised hope in the context of the amyloid cascade hypothesis of AD. NSAIDs such as R-flurbiprofen (or Tarenflurbil) have been tested in clinical trials. In young Tg2576 mice mimicking a prodromal stage of AD, chronic administration of R-flurbiprofen improved spatial learning with no significant effect on brain soluble A $\beta$ levels (Kukar et al., 2007). However, when administrated in Tg2576 mice at an advanced stage of the pathology, the drug decreased amyloid plaque load without inducing any spatial learning improvement. Thus, chronic administration of R-flurbiprofen might exert a preventive effect on AD progression (Kukar et al., 2007), but higher doses might be used to lower A $\beta 42$ at later stages of the pathology. However, given the gastrointestinal disturbances induced by NSAIDs, increasing the dose might become a concern. This dose issue was also pointed out in phase 1 of the clinical trial, where R-flurbiprofen, although tested at three different doses, had no effect on A $\beta 42$ levels of healthy subjects (Galasko et al., 2007). In phase 2, R-flurbiprofen demonstrated a beneficial dose-related effect on cognitive and functional outcomes in patients with mild AD (Wilcock et al., 2008). In phase 3, and despite the large cohort of AD patients involved, R-flurbiprofen did not reduce cognitive decline nor enhance patients' daily living activities (Green et al., 2009). More recently, a second generation of GSMs, including NGP 555, has been tested on young prodromal Tg2576 mice. Kounnas and colleagues reported that treatment with NGP 555 
induced spatial and working memory improvement and effectively lowered soluble plasmatic and cerebral A $\beta$ in these animals (Kounnas et al., 2017). Importantly, NGP 555 had no effect on Notch signaling nor other critical substrates, indicating a limited risk to induce cancer (Kounnas et al., 2017). In AD patients, a clinical trial in phase 1 was finalized but the results are not yet available (NCT02534480; https:// clinicaltrials.gov).

The overall inefficacy of anti-A $\beta$ and other drugs designed to reduce $A \beta$ in $A D$ patients has contributed to discredit the amyloid cascade hypothesis. In fact, the use of anti-A $\beta$ in mouse models of $A D$ resulted in substantial reduction in soluble $A \beta$ and amyloid plaques together with an improvement of cognitive functions in these animal models. However, when administrated to AD patients, these anti-A $\beta$ compounds successfully decreased amyloid plaque load but failed to enhance memory or cognitive functions. The fact that anti-A $\beta$ approaches succeed in limiting the progression of amyloid pathology without ameliorating cognitive function, points out the need to intervene at earlier stages of the disease. In this context, the identification of markers allowing early diagnosis of the pathology has become a priority.

\section{Alzheimer's Disease Patients Also Present Changes in Their Brain Activity}

In contrast to the apparent heterogeneity in the progression of amyloidopathy and cognitive deficits in $\mathrm{AD}$ patients, one phenomenon has proven to be remarkably consistent, particularly in the early stages of the disease: the alteration of the default mode network (DMN), as revealed by functional MRI (fMRI). This DMN corresponds to the activation of specific brain structures, including the precuneus, the posterior cingulate cortex, and the lateral and inferior parietal cortex, during inwardly oriented mental activity, such as introspection, mind wandering, and daydreaming. In contrast, this network is deactivated during outwardly mental tasks such as the acquisition and encoding of new information (Raichle et al., 2001). In fact, the task-induced deactivation of DMN components is critical for encoding and restitution of memory (Sperling et al., 2010). Hence, alteration of DMN activation and deactivation could participate to the cognitive deficits observed in AD patients. Indeed, fMRI studies revealed hippocampal hyperactivation and reduced deactivation of DMN during memory encoding in carriers of FAD-related mutations at presymptomatic stage (Quiroz et al., 2010), as well as in subjects with mild cognitive impairment (MCI) (Bakker et al., 2012). In contrast, during later stages of $\mathrm{AD}$, the hippocampal formation is hypoactive, deactivation of $\mathrm{DMN}$ components is reduced during learning, and the shift from "resting" to "active" mode is slower (Celone et al., 2006).

For long, hippocampal hyperactivation in prodromal AD and MCI patients has been interpreted as a compensatory mechanism to counteract emerging cognitive deficits (Putcha et al., 2011). Today, accumulating evidence indicates that this hippocampal hyperactivation is pathogenic by itself, and might directly impair learning and memory processes (Bakker et al., 2012). These observations led to the idea that this hyperactivity could reflect events of neuronal hypersynchrony. This hypersynchrony reflects excessive synchronization of activity of local neuronal populations, resulting in rhythmic discharges of large amplitude that can be observed on electroencephalographic (EEG) recordings (Fig. 2).

Tg2576



NTg

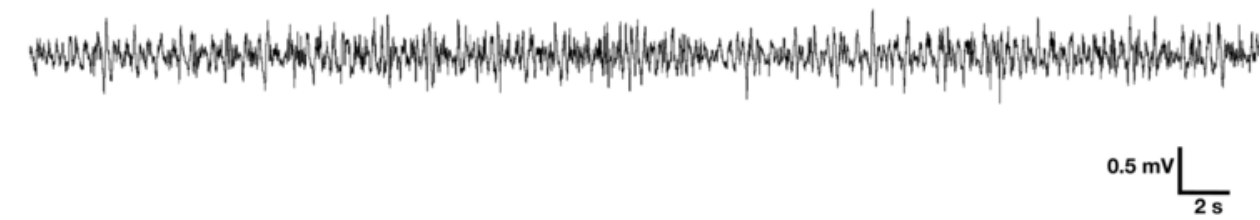

Fig. 2 Representative electroencephalographic (EEG) traces from a transgenic mouse modeling AD (Tg2576, top), and a nontransgenic mouse (NTg, bottom). On this EEG segment, the Tg2576 individual displays interictal spikes (black arrows), followed by a seizure (black line) characterized by high amplitude and high frequency oscillations lasting several seconds, followed by regular low-amplitude oscillations. 


\section{Hypersynchrony and Epilepsy in Alzheimer's Disease Mouse Models and Patients}

The modification of the DMN is only one of the many disturbances of brain activity in AD patients (Sperling et al., 2009). Indeed, AD condition is strongly associated with aberrant brain network activity, including hypersynchrony and epileptiform activity. In this context, mouse models of $\mathrm{AD}$ have proven to be highly relevant for the understanding of brain network abnormalities and neuronal dysfunction induced by amyloidopathy.

At first, epileptic phenotype was reported from opportunistic observations of tonic-clonic seizures or myoclonic movements in $\mathrm{AD}$ animals while running behavioral tests. For example, $41 \%$ of 24 -month-old APP23 mice exhibit tonico-clonic seizures, and $23 \%$ of them present myoclonic movements (Lalonde et al., 2005). Spontaneous epileptic seizures were also observed in the $\mathrm{Tg} 2576 \mathrm{mouse}$ line (Fig. 2), associated with aberrant behavior, such as repetitive movements, hyperactivity or myoclonic jerks (Hsiao et al., 1995) (Westmark et al., 2008). These reports were indeed strong cues that $\mathrm{AD}$ mice were experiencing dramatic modification of their brain activity.

The first EEG recordings providing indisputable evidence for epileptic activity in AD mouse models were conducted in the hAPPJ20 line that bears the Swedish and Indiana FAD-linked mutations of hAPP (Palop et al., 2007). These observations led to the idea that epileptic seizures and/or the remodeling of hippocampal networks induced by these aberrant activities may underlie AD-associated cognitive decline (Palop and Mucke, 2010). It also suggested that the epileptic phenotype was so far rather underestimated in mouse models of AD. Indeed, these findings encouraged longer EEG recordings, leading to the observation that 5-10\% of hAPPJ20 mice have at least one epileptic seizure over $24 \mathrm{~h}$ recording period (Verret et al., 2012). Following 2-weeks recording of 3-month-old APPswe/PS1dE9 mice, 25\% of the mice were found to display epileptic seizures; this proportion reached 55\% at the age of 4.5 months (Minkeviciene et al., 2009).

Apart from epileptic seizures, EEG recordings of AD mouse models have revealed the presence of epileptiform activity, called interictal spikes (when detected in individuals with seizures) or subclinical epileptiform activity (when detected in individuals without known seizures). These events are defined as paroxysmal sharp waveforms visible on EEG and lasting 20-200 ms, which disrupt background activity and are associated with a subsequent slow wave oscillations. So far, these epileptic events have been found in all mouse models of AD that have been submitted to EEG recording, including hAPPJ20 (Palop et al., 2007; Verret et al., 2012), Tg2576 (Bezzina et al., 2015; Kam et al., 2016) (Fig. 2), APPswe/PS1dE9 (Minkeviciene et al., 2009), and APP23 (Ittner et al., 2014). Interestingly, these epileptic events are observed as early as 5-6 weeks of age in Tg2576 mice (Bezzina et al., 2015; Kam et al., 2016). This observation prompted the field to consider detection of epileptiform activity in human subjects as a putative early marker for the disease.

Interestingly, early-onset $\mathrm{AD}$ in patients bearing FAD-linked mutations is well known to be associated with epileptic seizures (Larner and Doran, 2006; Cabrejo et al., 2006). In contrast, although the incidence of epileptic seizures in patients with late-onset AD is clearly higher than in age-matched controls (Amatniek et al., 2006), frank convulsive seizures are observed in only 5-20\% of patients with sporadic AD. Hence, the risk of seizures as compared to age-matched control population is higher in patients with familial AD than in sporadic AD (30-60 vs. 6-10 respectively) (Pandis and Scarmeas, 2012).

However, it is likely that the occurrence of complex partial seizures in sporadic AD is largely underestimated because long-term video-EEG monitoring and/or magnetoencephalography (MEG) are not routinely used in these patients. Indeed, a recent retrospective study revealed that in patients with video-EEG confirmation, complex partial seizures may represent the most common seizure type at early stage of AD (Vossel et al., 2013), highlighting the fact that this monitoring should be performed for accurate diagnosis of epilepsy in AD. To date, information regarding the occurrence of subclinical epileptiform activity in AD patients remains limited. Long-term video-EEG of 54 patients with MCI or AD revealed the presence of epileptiform activity (spikes or sharp waves) in $62 \%$ of patients already diagnosed with seizures, and in $6 \%$ of patients without detected seizures (Vossel et al., 2013). In a subsequent study performed by the same group, epileptiform activity was detected in $42.4 \%$ of AD patients (with or without previously detected epilepsy) (Vossel et al., 2016). Interestingly, these patients had no specific features (i.e., clinical, or demographic) that distinguished them from those without subclinical epileptiform activity. However, patients with interictal epileptic discharges had symptoms of cognitive decline 5.5 years earlier than patients without subclinical epileptiform activity (Vossel et al., 2016). This suggests that disease course is more aggressive in AD patients with subclinical epileptiform activity than in patients free of such brain network activity.

To summarize, seminal works using transgenic FAD mouse models have shown that increased A $\beta$ levels are sufficient to elicit epileptiform activity and seizures, even at early stages of the disease, and in the absence of neuronal loss. These observations indicate that imbalance between excitation and inhibition might be a robust hallmark of early stage $\mathrm{AD}$, and that cognitive decline might be, at least in part, related to aberrant brain activity. Importantly, studies using mouse models of AD have raised the possibility that epileptic seizures, and especially epileptiform activity, may remain largely underdiagnosed in AD patients. Today, screening for epileptiform activity is starting to be used in clinic for identifying patients who may decline more rapidly. Additionally, outcomes from long-term EEG and MEG/EEG recordings could enhance precision in future clinical trials. 


\section{Neuronal Mechanisms of Hypersynchrony/Epilepsy in Alzheimer's Disease Mouse Models}

Understanding the mechanisms behind the original alterations in neuronal network activities might be crucial to define the link between $A \beta$ overproduction and cognitive dysfunction. Furthermore, identifying the key players underlying these aberrant activities could provide new therapeutic targets.

Epileptic activity is well known to trigger inhibitory compensatory responses in the hippocampus, which may counteract the imbalance between excitation and inhibition, both in epileptic patients (Nagerl et al., 2000), and in animal models with hyperexcitability/epilepsy (Tonder et al., 1994). Although these compensatory inhibitory mechanisms may be efficiently reducing aberrant network hyperexcitability and hypersynchrony, they can also interfere with normal hippocampal network functions required for learning and memory processes (Palop et al., 2006). Interestingly, several of these modifications have also been observed in AD patients (Palop et al., 2003).

In $\mathrm{AD}$ mouse models, epileptiform activity is associated with alterations in several activity-related proteins in the dentate gyrus of the hippocampus. Specifically, a decrease in the expression of the calbindin protein involved in the regulation of calcium influx and neuronal excitability has been reported in several mouse lines such as hAPPJ20 (Palop et al., 2003, 2007), Tg2576 (Corbett et al., 2013; Krezymon et al., 2013; Verret et al., 2013), and APPswe/PS1dE9 (Minkeviciene et al., 2009). Strikingly, calbindin depletion has also been observed in the dentate gyrus of AD patients, with the most striking reductions seen in the most severely demented cases (Palop et al., 2003). Furthermore, some activity-dependent proteins known to be critical for synaptic plasticity and memory formation are down regulated in the hippocampus of mouse models of $\mathrm{AD}$, particularly in the granular layer of the dentate gyrus. For instance, learning-induced Fos expression is reduced in Tg2576 (Corbett et al., 2017), hAPPJ20 (Corbett et al., 2017; Palop et al., 2005), and TgCRND8 mice (Hamm et al., 2017). Another specific hallmark of compensatory remodeling of hippocampal circuits due to A $\beta$-induced epileptic activity is the sprouting of inhibitory axonal terminals in the molecular layer of the dentate gyrus (Palop et al., 2007; Verret et al., 2013). Noticeably, these alterations have been shown to correlate with each other and with learning and memory deficits (Palop et al., 2003), suggesting that these compensatory inhibitory responses could be causally linked to cognitive decline. Moreover, the reorganization of inhibitory hippocampal circuits prompted the field to study if and how populations of inhibitory neurons were affected by AD pathology.

Indeed, reduced numbers of parvalbumin- (PV) (Brady and Mufson, 1997) and somatostatin- (SOM) containing inhibitory interneurons (Davies et al., 1980) have been reported in postmortem brains of AD patients. The decrease of these GABAergic neuronal populations, which are strongly involved in the organization of brain activity (Cardin, 2018), is also observed in mouse models of the disease. For instance, SOM-expressing interneurons are reduced in the hippocampus of Tg2576 (Perez-Cruz et al., 2011) and TgCRND8 (Albuquerque et al., 2015) mice around 6 months of age. Moreover, PV-expressing interneurons are reduced as early as 6 months of age in the hippocampus of Tg2576 mice (Cattaud et al., 2018), and even at younger age (2 months) in TgCRND8 mice (Hamm et al., 2017).

PV cells are widely distributed and abundant GABAergic fast-spiking inhibitory interneurons able to synchronize the activity of large populations of excitatory neurons (Hu et al., 2014). As a result, they play major roles in regulating local ensemble activities, including theta and gamma oscillations. Interference with PV cell functioning in the hippocampus of wild-type mice leads to altered memory, illustrating their contribution to cognitive functions (Mann and Paulsen, 2007). Detailed investigations using AD mouse models have revealed functional impairment of PV interneurons. Indeed, PV cells have reduced perineuronal net (PNN) onto their soma and proximal neuritis in the hippocampus of prodromal (3-month-old) Tg2576 mice (Cattaud et al., 2018). This PV-specific form of extracellular matrix is involved in the stabilization of synapses onto PV cells, and loss of PNNs in the hippocampus is associated with epileptiform activity (Rankin-Gee et al., 2015). Hence, atypical PNN absence in AD condition may compromise the functioning of PV circuits and the associated cognitive controls such as memory retrieval (Favuzzi et al., 2017). Moreover, PV cells show reduced levels of voltage-gated sodium channel (VGSC) subunit Nav1.1 in hAPPJ20 (Verret et al., 2012), Tg2576 (Corbett et al., 2013), and TgCRND8 (Hamm et al., 2017) mice. These decreased Nav1.1 levels in PV cells, which can be found also in AD patients, are directly linked to altered network activity, i.e., depressed gamma oscillations, epileptiform activity, and memory deficits (Verret et al., 2012). Notably, restoring Nav1.1 level in hAPPJ20 mice by genetic manipulation (Verret et al., 2012), or transplantation of embryonic interneurons overexpressing Nav1.1 (Martinez-Losa et al., 2018), was sufficient to increase inhibitory synaptic activity and reduce network hypersynchrony. Thus, PV cells play a pivotal role in the disruption of inhibitory control in the AD brain, leading to major deficits in cognitive functions such as learning and memory.

\section{Using Antiepileptic Drugs Against Alzheimer's Disease?}

The aforementioned recent failure of clinical trials using anti-A $\beta$ compounds has prompted the field to consider alternative strategies to fight AD. The high prevalence of epileptic seizures and neuronal hyperexcitability in AD patients suggested that antiepileptic drugs (AEDs) could be used to treat the pathology.

Because of their direct influence on neuronal activity and subsequent glutamate release, VGSCs are the molecular targets of numerous AEDs. Among VGSC-blockers, phenytoin was first described in 1938 for efficiently reducing convulsive seizures both in epileptic patients and animal models of epilepsy (Bialer and White, 2010). Paradoxically, phenytoin exacerbates epileptiform activity in the hAPPJ20 mouse model of AD (Verret et al., 2012), but not in the APPswe/PS1dE9 mice (Ziyatdinova et al., 2011). The detrimental impact of phenytoin both on seizures and cognitive performance in hAPPJ20 mice might be linked to the reduced presence of Nav1.1 in 
PV interneurons. In this context, blocking Nav1.1 activity with phenytoin could reduce the activity of these Nav1.1-expressing inhibitory neurons and provoke the hyperexcitability of pyramidal neurons, resulting in exacerbation of hypersynchrony and epilepsy. Interestingly, a retrospective study of clinical data from $\mathrm{AD}$ and $\mathrm{MCI}$ patients who were treated with AEDs for diagnosed epilepsy revealed that phenytoin was less efficient for reducing epilepsy, and also less tolerated than other AEDs (Vossel et al., 2013).

The same study also revealed that levetiracetam (LEV) was among the most efficient AEDs for reducing epileptiform activity in MCI and $\mathrm{AD}$ patients, and that it was also well tolerated in this specific clinical context (Vossel et al., 2013). In contrast to phenytoin, LEV has proven to be beneficial for AD mouse models. Indeed, chronic LEV treatment reduces network hypersynchrony, synaptic deficits, and behavioral abnormalities in the hAPPJ20 mouse model (Sanchez et al., 2012). In addition, LEV injection for 30 days restores spatial memory in 7-month-old APPswe/PS1dE9 mice, associated with decreased A $\beta$ load and increased A $\beta$ clearance (Shi et al., 2013).

Furthermore, it has been shown that chronic treatment with low doses of LEV in amnestic MCI was able to reduce hippocampal hyperactivity and improve performance in hippocampal-dependent pattern separation task (Bakker et al., 2012). Additionally, a recent study compared the impact of low and high LEV doses on brain activity and cognitive performance in AD patients (Musaeus et al., 2017). Although no significant changes in cognitive performance were observed after a single dose of LEV, spectral analysis of EEG recordings revealed decreased coherence in the lower frequency bands $(1-4 \mathrm{~Hz})$ and increased coherence in the higher frequency bands $(13-30 \mathrm{~Hz})$. These subtle changes in brain oscillatory activity indicate that LEV might be beneficial for patients with AD (Musaeus et al., 2017). Noticeably, several clinical trials aiming at evaluating beneficial impact of LEV on brain activity and cognitive performance of AD patients are currently ongoing (NCT02002819 and NCT01554683, https://clinicaltrials.gov/).

\section{Alteration of Brain Oscillations in Alzheimer's Disease Mouse Models and Patients}

The brain relies on oscillatory rhythmic activity, generated by inhibitory interneurons, to organize information flow and to precisely time neuronal firing required for cognitive processing. The simultaneous unit activity of neuronal populations results in brain waves that can oscillate at different frequencies (in hertz-Hz) and whose power on the EEG reflects the behavioral state of the animal. Among brain rhythms, theta $(4-12 \mathrm{~Hz})$ and gamma $(40-150 \mathrm{~Hz})$ oscillations are involved in network stability, and both perceptual and memory processes.

Theta oscillations are considered critical for memory formation and synchronization between the hippocampus and several other brain structures (Cardin, 2018). Oscillations occurring at gamma frequencies are thought to transiently couple distributed networks that are processing related information. In human subjects, selective increase of gamma oscillations is observed during learning and predicts successful encoding of new memories (Sederberg et al., 2007). Similarly, in rodents, increased gamma activity (Yamamoto et al., 2014), and theta-gamma coupling (Tort et al., 2009) are induced during learning or novelty detection. Theta-gamma coupling reflects the nesting of gamma oscillations within the slower theta oscillations, and this comodulation might play a critical role in memory processes in the hippocampus. Noticeably, it is now known that both theta and gamma oscillatory activities are controlled by the synaptic activity of PV-expressing inhibitory fast-spiking interneurons (Cardin et al., 2009; Amilhon et al., 2015). As discussed above, PV cell function is strongly altered both in AD mouse models and patients. Altogether these observations led to the idea that AD-linked PV dysfunction causes abnormal brain oscillatory activity that might interfere with the intricate processes underlying cognitive deficits.

Indeed, mouse models of $\mathrm{AD}$, which are known to exhibit disrupted PV cells associated with hypersynchrony/epilepsy, are also experiencing abnormal brain oscillations. Abnormal fluctuations in the power of gamma oscillations have been observed in hAPPJ20 mice. Specifically, these mice exhibit brief peaks of increased gamma power and long periods of decreased gamma power, independently of their behavioral state (Verret et al., 2012). Similar abnormal fluctuation in behavior-induced gamma power occurs in 12-14-month-old Tg2576 mice (Cramer et al., 2012). Interestingly, TgCRND8 mice show aberrant oscillatory activity at prodromal stage, i.e., before detectable cognitive deficits and $A \beta$ accumulation. Specifically, although both transgenic and nontransgenic mice display increased theta power in the hippocampus following a sensorial stimulation, preplaque TgCRND8 mice show reduced gamma power on their EEG (Hamm et al., 2017). Furthermore, in vitro recordings from septo-hippocampal preparation of these mice revealed that theta-gamma coupling is impaired before detectable cognitive deficits and amyloidopathy (Goutagny et al., 2013). In the same line, a decrease of theta and gamma powers has been evidenced before disturbances of spatial learning in the 5xFAD mouse model (Schneider et al., 2014).

Characterization of aberrant brain oscillatory in AD mouse models at prodromal or early stage of the pathology has at least two important outcomes. First, the idea that EEG recording could be an easily implementable approach for a precocious detection of AD started to emerge in the field. Importantly, abnormal brain oscillatory activity has also been reported in AD patients and MCI. For instance, $\mathrm{AD}$ patients also show aberrant increases of sensory-evoked gamma power, which could be linked to decreased cortical inhibition (van Deursen et al., 2011). Overall, it is well admitted that AD patients typically present an increase in theta power associated with a decrease in relative power of gamma oscillations during resting state (Herrmann and Demiralp, 2005; Nimmrich et al., 2015). This amplitude of theta waves relatively to oscillatory activities of higher frequency has been proposed as an early marker of AD. Indeed, this measure allows the correct classification of $85 \%$ of MCI subjects, by making possible to distinguish the subjects who progress into $\mathrm{AD}$ from those who remain stable (Jelic et al., 2000). Interestingly, a longitudinal study revealed that subjects with subjective complaints had increased theta power 7 years before decline to $\mathrm{MCI}$ and/or conversion to dementia, highlighting the high sensitivity of EEG evaluations as predictors of future cognitive decline (Prichep et al., 2006). However, differences in baseline or resting state EEG can also be detected in other neurological conditions and might not be specific to AD (Herrmann and Demiralp, 2005). Hence, it has been proposed that recording EEG during behavioral tasks would provide higher specificity for AD condition (Hamm et al., 2015). Spatial disorientation is already present at $\mathrm{MCI}$ and early $\mathrm{AD}$ stages, and spatial navigation depends on theta and gamma oscillations. It has been 
shown that specific navigational tasks in both virtual and real-world paradigms could predict the risk of conversion from normal aging to MCI, and from MCI to dementia (Kalova et al., 2005; Weniger et al., 2011; Moodley et al., 2015). Therefore, the combination of spatial navigation tasks with EEG recordings may be useful for early and specific detection of AD.

Another major contribution of the study of brain oscillations in AD mouse models is that it promotes the idea that reducing PV cell-dependent aberrant oscillatory activity could contribute to the rescue of cognitive functions in AD condition (Palop and Mucke, 2016). Indeed, restoring PV cell function by overexpressing Nav1.1 is able to reduce gamma oscillations abnormalities, hypersynchrony, and cognitive functions in the hAPPJ20 mouse model of AD (Verret et al., 2012). In a follow-up study, the same group has recently demonstrated that grafting embryonic precursors of interneurons overexpressing Nav1.1 in the brain of adult hAPPJ20 mice induces the same beneficial effects, despite the presence of amyloidopathy (Martinez-Losa et al., 2018). Another study demonstrated that specific stimulation of PV cells at $40 \mathrm{~Hz}$ was sufficient to provoke the emergence of sustained gamma oscillations at this same frequency in the brain of 5xFAD mice (Iaccarino et al., 2016). Surprisingly, they also found that inducing gamma waves was initiating changes in gene expression that cause microglia to more efficiently clear extracellular $\mathrm{A} \beta$. In order to determine if these findings can have any therapeutic relevance, they aimed to induce gamma oscillations in a less-invasive way, and they submitted 5xFAD mice to $40-\mathrm{Hz}$ flickering lights. Strikingly, they observed that animals submitted to flickering light an hour a day over a week showed a $67 \%$ reduction of plaque load in the visual cortex, associated with reduced tau protein (Iaccarino et al., 2016). Although studies using mouse models of AD clearly demonstrate that promoting $40-\mathrm{Hz}$ oscillations has beneficial impacts in $\mathrm{AD}$ condition, whether the same effect will be seen in humans remains to be determined. Indeed, these findings opened the way for safety trials involving multi-sensory stimulation devices (such as glasses, headphones), sometimes associated with transcranial alternating current stimulation, that are able to induce gamma activity in different brain regions of AD patients (NCT03556280, NCT03661034, NCT03543878, NCT03657745, and NCT02663531; https://clinicaltrials.gov/ ).

\section{Conclusion}

Mouse models of AD do not perfectly mimic the disorder, and many therapies that appeared promising in rodents have failed in humans. However, animal models are valuable tools for understanding how AD pathology affects neuronal activity at multiple levels of brain function, and how synaptic, cellular, and network changes trigger cognitive impairment. Experimental data from mouse models of AD continue to provide new knowledge about the pathology, both by allowing the identification of molecular targets pivotal to the disease process and by characterizing biomarkers that can be used for early diagnosis in patients.

\section{References}

Albuquerque, M.S., Mahar, I., Davoli, M.A., et al., 2015. Regional and sub-regional differences in hippocampal GABAergic neuronal vulnerability in the TgCRND8 mouse model of Alzheimer's disease. Frontiers in Aging Neuroscience 7, 30.

Amatniek, J.C., Hauser, W.A., DelCastillo-Castaneda, C., et al., 2006. Incidence and predictors of seizures in patients with Alzheimer's disease. Epilepsia 47 (5), 867-872.

Amilhon, B., Huh, C.Y., Manseau, F., et al., 2015. Parvalbumin Interneurons of hippocampus tune population activity at theta frequency. Neuron 86 (5), 1277-1289.

Bakker, A., Krauss, G.L., Albert, M.S., et al., 2012. Reduction of hippocampal hyperactivity improves cognition in amnestic mild cognitive impairment. Neuron 74 (3), 467-474.

Bayer, A.J., Bullock, R., Jones, R.W., et al., 2005. Evaluation of the safety and immunogenicity of synthetic Abeta42 (AN1792) in patients with AD. Neurology 64 (1), 94-101.

Bezzina, C., Verret, L., Juan, C., et al., 2015. Early onset of hypersynchronous network activity and expression of a marker of chronic seizures in the Tg2576 mouse model of Alzheimer's disease. PLoS One 10 (3), e0119910.

Bialer, M., White, H.S., 2010. Key factors in the discovery and development of new antiepileptic drugs. Nature Reviews. Drug Discovery 9 (1), 68-82.

Brady, D.R., Mufson, E.J., 1997. Parvalbumin-immunoreactive neurons in the hippocampal formation of Alzheimer's diseased brain. Neuroscience 80 (4), $1113-1125$.

Cabrejo, L., Guyant-Marechal, L., Laquerriere, A., et al., 2006. Phenotype associated with APP duplication in five families. Brain 129 (Pt 11), $2966-2976$.

Cardin, J.A., 2018. Inhibitory interneurons regulate temporal precision and correlations in cortical circuits. Trends in Neurosciences 41 (10), 689-700.

Cardin, J.A., Carlen, M., Meletis, K., et al., 2009. Driving fast-spiking cells induces gamma rhythm and controls sensory responses. Nature 459 (7247), 663-667.

Cattaud, V., Bezzina, C., Rey, C.C., et al., 2018. Early disruption of parvalbumin expression and perineuronal nets in the hippocampus of the Tg2576 mouse model of Alzheimer's disease can be rescued by enriched environment. Neurobiology of Aging 72, 147-158.

Celone, K.A., Calhoun, V.D., Dickerson, B.C., et al., 2006. Alterations in memory networks in mild cognitive impairment and Alzheimer's disease: An independent component analysis. The Journal of Neuroscience 26 (40), 10222-10231.

Corbett, B.F., Leiser, S.C., Ling, H.P., et al., 2013. Sodium channel cleavage is associated with aberrant neuronal activity and cognitive deficits in a mouse model of Alzheimer's disease. The Journal of Neuroscience 33 (16), 7020-7026.

Corbett, B.F., You, J.C., Zhang, X., et al., 2017. DeltaFosB regulates gene expression and cognitive dysfunction in a mouse model of Alzheimer's disease. Cell Reports 20 (2), 344-355.

Cramer, P.E., Cirrito, J.R., Wesson, D.W., et al., 2012. ApoE-directed therapeutics rapidly clear beta-amyloid and reverse deficits in AD mouse models. Science 335 (6075), 1503-1506.

Das, P., Verbeeck, C., Minter, L., et al., 2012. Transient pharmacologic lowering of Abeta production prior to deposition results in sustained reduction of amyloid plaque pathology. Molecular Neurodegeneration 7, 39.

Daumas, S., Sandin, J., Chen, K.S., et al., 2008. Faster forgetting contributes to impaired spatial memory in the PDAPP mouse: Deficit in memory retrieval associated with increased sensitivity to interference?. Learning \& Memory 15 (9), 625-632. 
Davies, P., Katzman, R., Terry, R.D., 1980. Reduced somatostatin-like immunoreactivity in cerebral cortex from cases of Alzheimer disease and Alzheimer senile dementa. Nature 288 (5788), 279-280.

Dodart, J.C., Meziane, H., Mathis, C., et al., 1999. Behavioral disturbances in transgenic mice overexpressing the V717F beta-amyloid precursor protein. Behavioral Neuroscience 113 (5), 982-990.

Doody, R.S., Raman, R., Farlow, M., et al., 2013. A phase 3 trial of semagacestat for treatment of Alzheimer's disease. The New England Journal of Medicine $369(4), 341-350$.

Favuzzi, E., Marques-Smith, A., Deogracias, R., et al., 2017. Activity-dependent gating of Parvalbumin interneuron function by the Perineuronal net protein Brevican. Neuron 95 (3), 639-55.e10.

Galasko, D.R., Graff-Radford, N., May, S., et al., 2007. Safety, tolerability, pharmacokinetics, and Abeta levels after short-term administration of R-flurbiprofen in healthy elderly individuals. Alzheimer Disease and Associated Disorders 21 (4), 292-299.

Games, D., Adams, D., Alessandrini, R., et al., 1995. Alzheimer-type neuropathology in transgenic mice overexpressing V717F beta-amyloid precursor protein. Nature 373 (6514), 523-527.

Garcia-Alloza, M., Subramanian, M., Thyssen, D., et al., 2009. Existing plaques and neuritic abnormalities in APP:PS1 mice are not affected by administration of the gamma-secretase inhibitor LY-411575. Molecular Neurodegeneration 4, 19.

Gotz, J., Bodea, L.G., Goedert, M., 2018. Alzheimer disease rodent models for Alzheimer disease. Nature Reviews Neuroscience 19 (10), 583-598.

Goutagny, R., Gu, N., Cavanagh, C., et al., 2013. Alterations in hippocampal network oscillations and theta-gamma coupling arise before Abeta overproduction in a mouse model of Alzheimer's disease. The European Journal of Neuroscience 37 (12), 1896-1902.

Green, R.C., Schneider, L.S., Amato, D.A., et al., 2009. Effect of tarenflurbil on cognitive decline and activities of daily living in patients with mild Alzheimer disease: A randomized controlled trial. Journal of the American Medical Association 302 (23), 2557-2564.

Hamm, V., Heraud, C., Cassel, J.C., et al., 2015. Precocious alterations of brain oscillatory activity in Alzheimer's disease: A window of opportunity for early diagnosis and treatment. Frontiers in Cellular Neuroscience 9, 491.

Hamm, V., Heraud, C., Bott, J.B., et al., 2017. Differential contribution of APP metabolites to early cognitive deficits in a TgCRND8 mouse model of Alzheimer's disease. Science Advances 3 (2), e1601068.

Hardy, J.A., Higgins, G.A., 1992. Alzheimer's disease: The amyloid cascade hypothesis. Science 256 (5054), 184-185.

Harris, J.A., Devidze, N., Halabisky, B., et al., 2010. Many neuronal and behavioral impairments in transgenic mouse models of Alzheimer's disease are independent of caspase cleavage of the amyloid precursor protein. The Journal of Neuroscience 30 (1), 372-381.

Herrmann, C.S., Demiralp, T., 2005. Human EEG gamma oscillations in neuropsychiatric disorders. Clinical Neurophysiology 116 (12), $2719-2733$

Holmes, C., Boche, D., Wilkinson, D., et al., 2008. Long-term effects of Abeta42 immunisation in Alzheimer's disease: Follow-up of a randomised, placebo-controlled phase I trial. Lancet 372 (9634), 216-223.

Hsiao, K.K., Borchelt, D.R., Olson, K., et al., 1995. Age-related CNS disorder and early death in transgenic FVB/N mice overexpressing Alzheimer amyloid precursor proteins. Neuron $15(5), 1203-1218$.

Hu, H., Gan, J., Jonas, P., 2014. Interneurons. Fast-spiking, parvalbumin(+) GABAergic interneurons: From cellular design to microcircuit function. Science 345 (6196), 1255263

Iaccarino, H.F., Singer, A.C., Martorell, A.J., et al., 2016. Gamma frequency entrainment attenuates amyloid load and modifies microglia. Nature 540 (7632), 230-235.

Ittner, A.A., Gladbach, A., Bertz, J., et al., 2014. p38 MAP kinase-mediated NMDA receptor-dependent suppression of hippocampal hypersynchronicity in a mouse model of Alzheimer's disease. Acta Neuropathologica Communications 2, 149.

Jankowsky, J.L., Slunt, H.H., Ratovitski, T., et al., 2001. Co-expression of multiple transgenes in mouse CNS: A comparison of strategies. Biomolecular Engineering 17 (6), 157-165.

Jankowsky, J.L., Fadale, D.J., Anderson, J., et al., 2004. Mutant presenilins specifically elevate the levels of the 42 residue beta-amyloid peptide in vivo: Evidence for augmentation of a 42 -specific gamma secretase. Human Molecular Genetics 13 (2), 159-170.

Jankowsky, J.L., Younkin, L.H., Gonzales, V., et al., 2007. Rodent a beta modulates the solubility and distribution of amyloid deposits in transgenic mice. The Journal of Biological Chemistry 282 (31), 22707-22720.

Janus, C., Pearson, J., McLaurin, J., et al., 2000. A beta peptide immunization reduces behavioral impairment and plaques in a model of Alzheimer's disease. Nature 408 (6815), 979-982.

Jelic, V., Johansson, S.E., Almkvist, O., et al., 2000. Quantitative electroencephalography in mild cognitive impairment: Longitudinal changes and possible prediction of Alzheimer's disease. Neurobiology of Aging 21 (4), 533-540.

Kalova, E., Vlcek, K., Jarolimova, E., Bures, J., 2005. Allothetic orientation and sequential ordering of places is impaired in early stages of Alzheimer's disease: Corresponding results in real space tests and computer tests. Behavioural Brain Research 159 (2), 175-186.

Kam, K., Duffy, A.M., Moretto, J., et al., 2016. Interictal spikes during sleep are an early defect in the Tg2576 mouse model of beta-amyloid neuropathology. Scientific Reports 6, 20119.

Kawarabayashi, T., Younkin, L.H., Saido, T.C., et al., 2001. Age-dependent changes in brain, CSF, and plasma amyloid (beta) protein in the Tg2576 transgenic mouse model of Alzheimer's disease. The Journal of Neuroscience 21 (2), 372-381.

King, D.L., Arendash, G.W., Crawford, F., et al., 1999. Progressive and gender-dependent cognitive impairment in the APP(SW) transgenic mouse model for Alzheimer's disease. Behavioural Brain Research 103 (2), 145-162.

Kopan, R., Ilagan, M.X., 2009. The canonical notch signaling pathway: Unfolding the activation mechanism. Cell 137 (2), $216-233$.

Kounnas, M.Z., Lane-Donovan, C., Nowakowski, D.W., et al., 2017. NGP 555, a gamma-Secretase modulator, lowers the amyloid biomarker, Abeta42, in cerebrospinal fluid while preventing Alzheimer's disease cognitive decline in rodents. Alzheimers Dement (N Y). 3 (1), 65-73.

Krezymon, A., Richetin, K., Halley, H., et al., 2013. Modifications of hippocampal circuits and early disruption of adult neurogenesis in the tg2576 mouse model of Alzheimer's disease. PLoS One 8 (9), e76497.

Kukar, T., Prescott, S., Eriksen, J.L., et al., 2007. Chronic administration of R-flurbiprofen attenuates learning impairments in transgenic amyloid precursor protein mice. BMC Neuroscience 8,54 .

Lalonde, R., Dumont, M., Staufenbiel, M., Strazielle, C., 2005. Neurobehavioral characterization of APP23 transgenic mice with the SHIRPA primary screen. Behavioural Brain Research 157 (1), 91-98.

Larner, A.J., Doran, M., 2006. Clinical phenotypic heterogeneity of Alzheimer's disease associated with mutations of the presenilin-1 gene. Journal of Neurology 253 (2), 139-158.

Lesne, S., Koh, M.T., Kotilinek, L., et al., 2006. A specific amyloid-beta protein assembly in the brain impairs memory. Nature 440 (7082), 352-357.

Mann, E.O., Paulsen, O., 2007. Role of GABAergic inhibition in hippocampal network oscillations. Trends in Neurosciences 30 (7), 343-349.

Martinez-Losa, M., Tracy, T.E., Ma, K., et al., 2018. Nav1.1-overexpressing interneuron transplants restore brain rhythms and cognition in a mouse model of Alzheimer's disease. Neuron 98 (1), 75-89.e5.

Minkeviciene, R., Rheims, S., Dobszay, M.B., et al., 2009. Amyloid beta-induced neuronal hyperexcitability triggers progressive epilepsy. The Journal of Neuroscience 29 (11), 3453-3462. 
Moodley, K., Minati, L., Contarino, V., et al., 2015. Diagnostic differentiation of mild cognitive impairment due to Alzheimer's disease using a hippocampus-dependent test of spatial memory. Hippocampus 25 (8), 939-951.

Morgan, D., Diamond, D.M., Gottschall, P.E., et al., 2000. A beta peptide vaccination prevents memory loss in an animal model of Alzheimer's disease. Nature 408 (6815), 982-985.

Mucke, L., Masliah, E., Yu, G.Q., et al., 2000. High-level neuronal expression of abeta 1-42 in wild-type human amyloid protein precursor transgenic mice: Synaptotoxicity without plaque formation. The Journal of Neuroscience 20 (11), 4050-4058.

Musaeus, C.S., Shafi, M.M., Santarnecchi, E., et al., 2017. Levetiracetam alters oscillatory connectivity in Alzheimer's disease. Journal of Alzheimer's Disease 58 (4), 1065-1076.

Nagerl, U.V., Mody, I., Jeub, M., et al., 2000. Surviving granule cells of the sclerotic human hippocampus have reduced Ca( $2+)$ influx because of a loss of calbindin-D(28k) in temporal lobe epilepsy. The Journal of Neuroscience 20 (5), 1831-1836.

Nimmrich, V., Draguhn, A., Axmacher, N., 2015. Neuronal network oscillations in neurodegenerative diseases. Neuromolecular Medicine 17 (3), $270-284$.

Orgogozo, J.M., Gilman, S., Dartigues, J.F., et al., 2003. Subacute meningoencephalitis in a subset of patients with AD after Abeta42 immunization. Neurology 61 (1), 46-54.

Palop, J.J., Mucke, L., 2010. Amyloid-beta-induced neuronal dysfunction in Alzheimer's disease: From synapses toward neural networks. Nature Neuroscience 13 (7), 812-818.

Palop, J.J., Mucke, L., 2016. Network abnormalities and interneuron dysfunction in Alzheimer disease. Nature Reviews. Neuroscience 17 (12), $777-792$.

Palop, J.J., Jones, B., Kekonius, L., et al., 2003. Neuronal depletion of calcium-dependent proteins in the dentate gyrus is tightly linked to Alzheimer's disease-related cognitive deficits. Proceedings of the National Academy of Sciences of the United States of America 100 (16), 9572-9577.

Palop, J.J., Chin, J., Bien-Ly, N., et al., 2005. Vulnerability of dentate granule cells to disruption of arc expression in human amyloid precursor protein transgenic mice. The Journal of Neuroscience 25 (42), 9686-9693.

Palop, J.J., Chin, J., Mucke, L., 2006. A network dysfunction perspective on neurodegenerative diseases. Nature 443 (7113), $768-773$.

Palop, J.J., Chin, J., Roberson, E.D., et al., 2007. Aberrant excitatory neuronal activity and compensatory remodeling of inhibitory hippocampal circuits in mouse models of Alzheimer's disease. Neuron 55 (5), 697-711.

Pandis, D., Scarmeas, N., 2012. Seizures in Alzheimer disease: Clinical and epidemiological data. Epilepsy Currents 12 (5), $184-187$.

Perez-Cruz, C., Nolte, M.W., van Gaalen, M.M., et al., 2011. Reduced spine density in specific regions of CA1 pyramidal neurons in two transgenic mouse models of Alzheimer's disease. The Journal of Neuroscience 31 (10), 3926-3934.

Prichep, L.S., John, E.R., Ferris, S.H., et al., 2006. Prediction of longitudinal cognitive decline in normal elderly with subjective complaints using electrophysiological imaging. Neurobiology of Aging 27 (3), 471-481.

Putcha, D., Brickhouse, M., O'Keefe, K., et al., 2011. Hippocampal hyperactivation associated with cortical thinning in Alzheimer's disease signature regions in non-demented elderly adults. The Journal of Neuroscience 31 (48), 17680-17688.

Quiroz, Y.T., Budson, A.E., Celone, K., et al., 2010. Hippocampal hyperactivation in presymptomatic familial Alzheimer's disease. Annals of Neurology 68 (6), 865-875.

Raichle, M.E., MacLeod, A.M., Snyder, A.Z., et al., 2001. A default mode of brain function. Proceedings of the National Academy of Sciences of the United States of America 98 (2), 676-682.

Rankin-Gee, E.K., McRae, P.A., Baranov, E., et al., 2015. Perineuronal net degradation in epilepsy. Epilepsia 56 (7), $1124-1133$.

Sanchez, P.E., Zhu, L., Verret, L., et al., 2012. Levetiracetam suppresses neuronal network dysfunction and reverses synaptic and cognitive deficits in an Alzheimer's disease model. Proceedings of the National Academy of Sciences of the United States of America 109 (42), E2895-E2903.

Schenk, D., Barbour, R., Dunn, W., et al., 1999. Immunization with amyloid-beta attenuates Alzheimer-disease-like pathology in the PDAPP mouse. Nature 400 (6740), 173-177.

Schneider, F., Baldauf, K., Wetzel, W., Reymann, K.G., 2014. Behavioral and EEG changes in male 5xFAD mice. Physiology \& Behavior $135,25-33$.

Sederberg, P.B., Schulze-Bonhage, A., Madsen, J.R., et al., 2007. Hippocampal and neocortical gamma oscillations predict memory formation in humans. Cerebral Cortex 17 (5), 1190-1196.

Sevigny, J., Chiao, P., Bussiere, T., et al., 2016. The antibody aducanumab reduces Abeta plaques in Alzheimer's disease. Nature 537 (7618), $50-56$.

Shi, J.Q., Wang, B.R., Tian, Y.Y., et al., 2013. Antiepileptics topiramate and levetiracetam alleviate behavioral deficits and reduce neuropathology in APPswe/ PS1dE9 transgenic mice. CNS Neuroscience \& Therapeutics 19 (11), 871-881.

Sperling, R.A., Laviolette, P.S., O'Keefe, K., et al., 2009. Amyloid deposition is associated with impaired default network function in older persons without dementia. Neuron 63 (2), 178-188.

Sperling, R.A., Dickerson, B.C., Pihlajamaki, M., et al., 2010. Functional alterations in memory networks in early Alzheimer's disease. Neuromolecular Medicine 12 (1), 27-43.

Tonder, N., Kragh, J., Finsen, B.R., et al., 1994. Kindling induces transient changes in neuronal expression of somatostatin, neuropeptide Y, and calbindin in adult rat hippocampus and fascia dentata. Epilepsia 35 (6), 1299-1308.

Tort, A.B., Komorowski, R.W., Manns, J.R., et al., 2009. Theta-gamma coupling increases during the learning of item-context associations. Proceedings of the National Academy of Sciences of the United States of America 106 (49), 20942-20947.

van Deursen, J.A., Vuurman, E.F., van Kranen-Mastenbroek, V.H., et al., 2011. 40-Hz steady state response in Alzheimer's disease and mild cognitive impairment. Neurobiology of Aging 32 (1), 24-30.

Vegh, M.J., Heldring, C.M., Kamphuis, W., et al., 2014. Reducing hippocampal extracellular matrix reverses early memory deficits in a mouse model of Alzheimer's disease. Acta Neuropathologica Communications 2, 76.

Vellas, B., Black, R., Thal, L.J., et al., 2009. Long-term follow-up of patients immunized with AN1792: Reduced functional decline in antibody responders. Current Alzheimer Research 6 (2), 144-151.

Verret, L., Mann, E.O., Hang, G.B., et al., 2012. Inhibitory interneuron deficit links altered network activity and cognitive dysfunction in Alzheimer model. Cell 149 (3), 708-721.

Verret, L., Krezymon, A., Halley, H., et al., 2013. Transient enriched housing before amyloidosis onset sustains cognitive improvement in Tg2576 mice. Neurobiology of Aging 34 (1), 211-225.

Vetrivel, K.S., Zhang, Y.W., Xu, H., Thinakaran, G., 2006. Pathological and physiological functions of presenilins. Molecular Neurodegeneration 1, 4.

Vossel, K.A., Beagle, A.J., Rabinovici, G.D., et al., 2013. Seizures and epileptiform activity in the early stages of Alzheimer disease. JAMA Neurology 70 (9), 1158-1166.

Vossel, K.A., Ranasinghe, K.G., Beagle, A.J., et al., 2016. Incidence and impact of subclinical epileptiform activity in Alzheimer's disease. Annals of Neurology 80 (6), 858-870.

Weggen, S., Eriksen, J.L., Sagi, S.A., et al., 2003. Abeta42-lowering nonsteroidal anti-inflammatory drugs preserve intramembrane cleavage of the amyloid precursor protein (APP) and ErbB-4 receptor and signaling through the APP intracellular domain. The Journal of Biological Chemistry 278 (33), 30748-30754.

Weniger, G., Ruhleder, M., Lange, C., et al., 2011. Egocentric and allocentric memory as assessed by virtual reality in individuals with amnestic mild cognitive impairment. Neuropsychologia 49 (3), 518-527. 
Westerman, M.A., Cooper-Blacketer, D., Mariash, A., et al., 2002. The relationship between Abeta and memory in the Tg2576 mouse model of Alzheimer's disease. The Journal of Neuroscience 22 (5), 1858-1867.

Westmark, C.J., Westmark, P.R., Beard, A.M., et al., 2008. Seizure susceptibility and mortality in mice that over-express amyloid precursor protein. International Journal of Clinical and Experimental Pathology 1 (2), 157-168.

Wilcock, G.K., Black, S.E., Hendrix, S.B., et al., 2008. Efficacy and safety of tarenflurbil in mild to moderate Alzheimer's disease: A randomised phase II trial. Lancet Neurology 7 (6), 483-493.

Yamamoto, J., Suh, J., Takeuchi, D., Tonegawa, S., 2014. Successful execution of working memory linked to synchronized high-frequency gamma oscillations. Cell 157 (4), 845-857.

Ziyatdinova, S., Gurevicius, K., Kutchiashvili, N., et al., 2011. Spontaneous epileptiform discharges in a mouse model of Alzheimer's disease are suppressed by antiepileptic drugs that block sodium channels. Epilepsy Research 94 (1-2), 75-85.

\section{Further Reading}

Born, H.A., 2015. Seizures in Alzheimer's disease. Neuroscience 286, 251-263.

Buzsaki, G., 2002. Theta oscillations in the hippocampus. Neuron 33 (3), 325-340.

Jankowsky, J.L., Zheng, H., 2017. Practical considerations for choosing a mouse model of Alzheimer's disease. Molecular Neurodegeneration 12 (1), 89.

McDermott, B., Porter, E., Hughes, D., et al., 2018. Gamma band neural stimulation in humans and the promise of a new modality to prevent and treat Alzheimer's disease. Journal of Alzheimer's Disease 65 (2), 363-392.

Nicastro, N., Assal, F., Seeck, M., 2016. From here to epilepsy: The risk of seizure in patients with Alzheimer's disease. Epileptic Disorders 18 (1), 1-12. Querfurth, H.W., LaFerla, F.M., 2010. Alzheimer's disease. The New England Journal of Medicine 362 (4), 329-344.

Reitz, C., 2012. Alzheimer's disease and the amyloid cascade hypothesis: A critical review. International Journal of Alzheimer's Disease $2012,369808$.

Tang, Y.P., Gershon, E.S., 2003. Genetic studies in Alzheimer's disease. Dialogues in Clinical Neuroscience 5 (1), 17-26.

Verret, L., Rampon, C., 2013. Amyloidogenesis, neurogenesis, learning, and memory in Alzheimer's disease: Lessons from transgenic mouse models. In: Derreumaux, P. (Ed.), Alzheimer's disease: Insights into low molecular weight and cytotoxic aggregates from computer simulations. Imperial College Press.

Wang, D., Fawcett, J., 2012. The perineuronal net and the control of CNS plasticity. Cell and Tissue Research 349 (1), 147-160.

\section{Relevant Websites}

https://www.alzforum.org/research-models:alzheimers-disease. https://clinicaltrials.gov. 